\title{
A PRELIMINARY INVESTIGATION OF PERIOD CHANGES \\ FOR W VIRGINIS STARS IN GLOBULAR CLUSTERS
}

\section{CHRISTINE M. COUTTS}

David Dunlap Observatory, University of Toronto, Richmond Hill, Ontario, Canada,

Investigations in recent years have shown that there may be two mechanisms which place stars in the W Virginis instability region (Kraft, 1972). The variables with periods less than 8 days seem to be in the stage of 'above horizontal branch' evolution discussed by Strom et al. (1970). The longer period group apparently results when thermal instabilities in the helium burning shell of an asymptotic red giant branch star cause it to loop to the left in the HR diagram. This longer period group has been investigated by Schwarzschild and Härm (1970) and Mengel (1972). The present study has been undertaken to see if there are any notable differences between the period changes of variables belonging to the two groups.

Period changes for the variables in M13 have already been discussed by Osborn (1969). The periods of the W Virginis stars in M5 have been studied by Coutts and

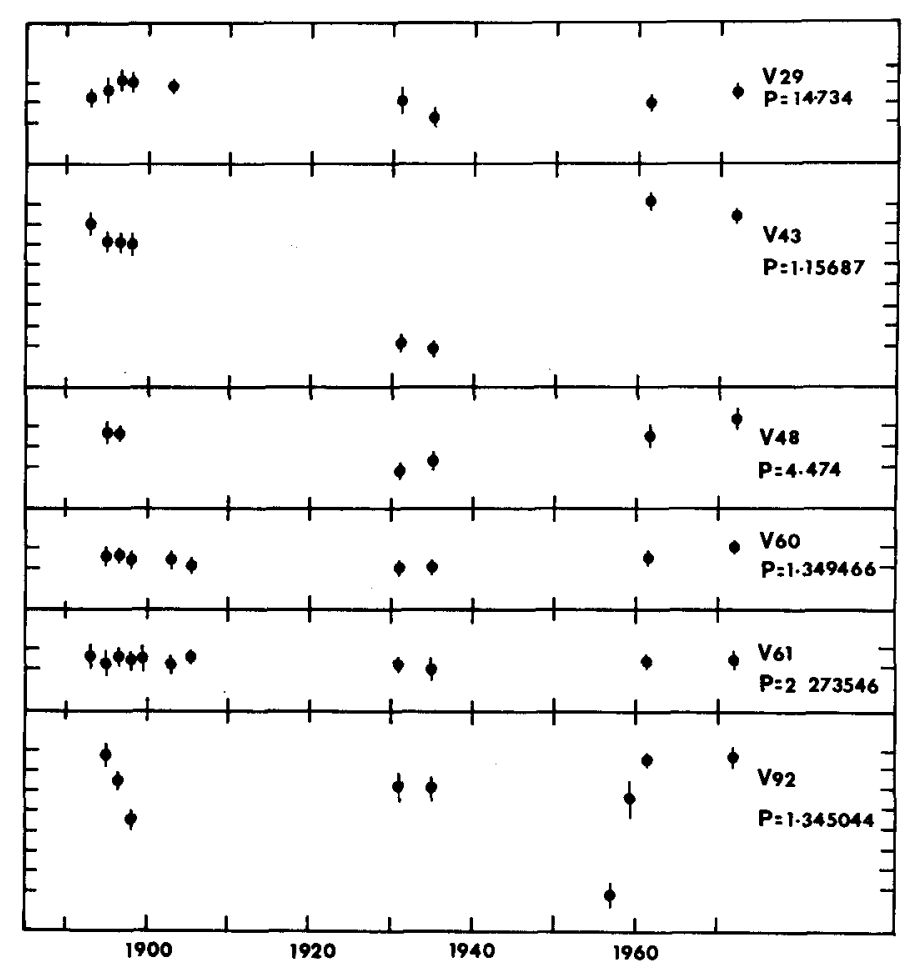

Fig. 1. Phase-shift diagrams for the variables in $\omega$ Centauri. The spacing of the marks on the vertical axis is 0.2 of the period. The date in years is indicated along the horizontal axis. Error bars indicate probable errors. 
Sawyer Hogg (1972a, 1972b). In the present investigation, phase-shift diagrams have been plotted for the W Virginis stars in three other clusters: $\omega$ Centauri, M14 and M2.

The material for $\omega$ Centauri has been taken from the published work of Bailey (1902), Sawyer (1931), Martin (1938), Eggen (1961), Dickens and Carey (1967), and Wilkens (1967). In addition, the author obtained a series of photographs of $\omega$ Centauri with the David Dunlap 24-in. telescope at Cerro Las Campanas, Chile in May and July, 1972. These phase-shift diagrams are shown in Figure 1. The diagrams for M14 (Figure 2) are based on the material published by Sawyer Hogg and Wehlau (1966, 1968), and Demers and Wehlau (1971). Those for M2 (Figure 3) are from Sawyer (1935) and from Poole (1968), a Master's thesis based on Sawyer Hogg's collection of plates at the David Dunlap Observatory.

Table I summarizes the results of the investigations of period changes of W Vir-

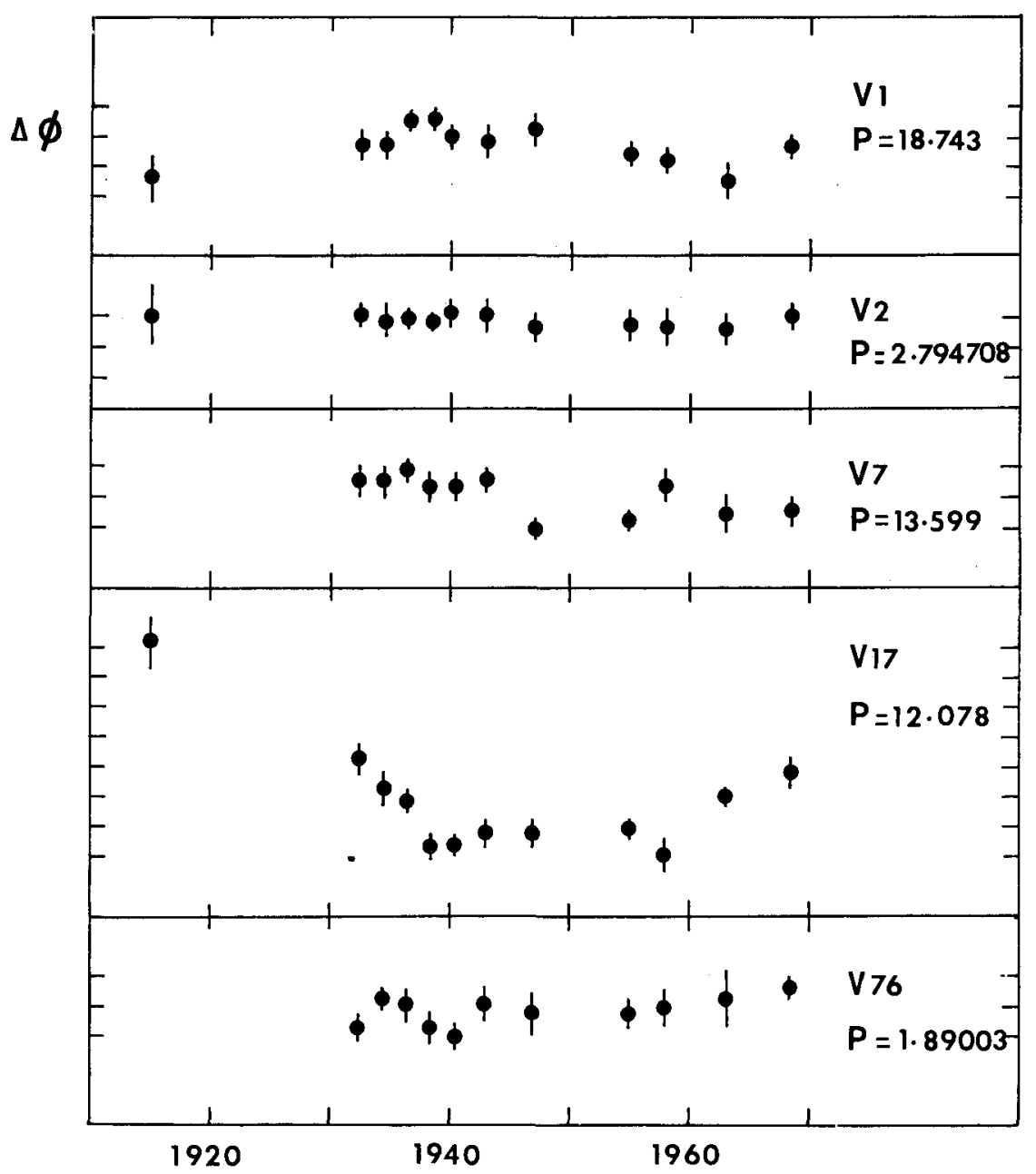

Fig. 2. Phase-shift diagrams for the variables in M14. 


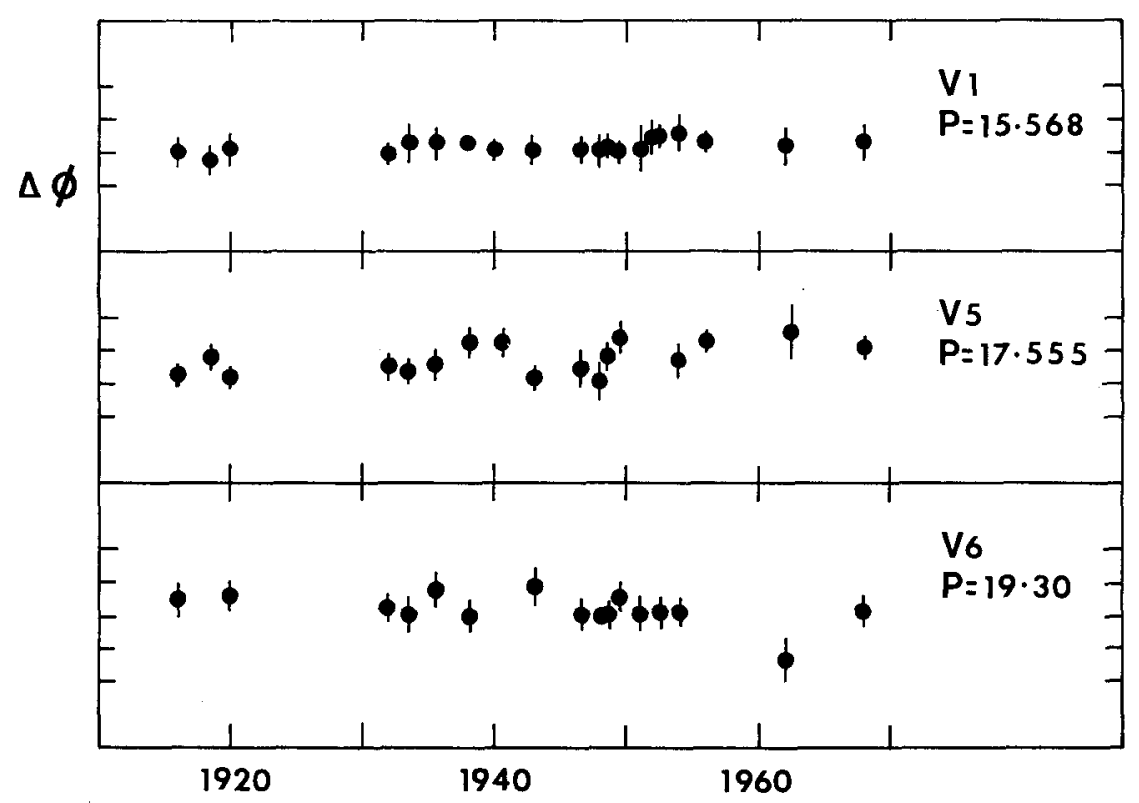

Fig. 3. Phase-shift diagrams for the variables in M2.

TABLE I

Summary of the period changes

\begin{tabular}{|c|c|c|c|}
\hline Cluster & Variable & Period & $\begin{array}{l}\text { Remarks about } \\
\text { period change }\end{array}$ \\
\hline \multirow[t]{6}{*}{$\omega \mathrm{Cen}$} & 29 & $14 \mathrm{~d} 734$ & small fluctuations \\
\hline & 43 & 1.15687 & fluctuations \\
\hline & 48 & 4.474 & increasing \\
\hline & 60 & 1.349466 & increasing \\
\hline & 61 & 2.273546 & constant \\
\hline & 92 & 1.345044 & fluctuations \\
\hline \multirow[t]{2}{*}{ M5 } & 42 & 25.738 & constant \\
\hline & 84 & 26.42 & fluctuations \\
\hline \multirow[t]{3}{*}{ M13 } & 1 & 1.458997 & probably constant \\
\hline & 2 & 5.110939 & increasing \\
\hline & 6 & 2.112867 & probably constant \\
\hline \multirow[t]{5}{*}{ M14 } & 1 & 18.743 & fluctuations \\
\hline & 2 & 2.794708 & constant \\
\hline & 7 & 13.599 & fluctuations \\
\hline & 17 & 12.078 & increasing \\
\hline & 76 & 1.89003 & probably constant \\
\hline \multirow[t]{3}{*}{ M2 } & 1 & 15.568 & constant \\
\hline & 5 & 17.555 & constant \\
\hline & 6 & 19.30 & small fluctuations \\
\hline
\end{tabular}


ginis stars in the five globular clusters, $\omega$ Cen, M5, M13, M14 and M2. Of the 19 stars studied, 10 have periods less than 8 days and 9 have periods longer than 8 days. In the shorter period group, 5 have constant periods, 3 have increasing periods and 2 have periods which have fluctuated during the last 60 years (approximately). (If the phase-shift diagram can be fit by a straight line, we have assumed that the period has remained constant over the interval of observations. This is not necessarily the case). In the longer period group, 3 have constant periods, 1 has an increasing period and 5 have periods which have fluctuated.

It seems that among the short period group, there is a greater tendency for the periods to remain constant or to increase, while the variables with longer periods have periods which fluctuate. The minimum detectable period change in 60 years depends on the period itself and is given by $\triangle P=10^{-5} P$. Thus the shorter the period, the smaller the period change that can be detected. It may therefore be significant that half of the short period stars studied have constant periods, while over half of the longer period variables have fluctuating periods. At this point, it is difficult to conclude anything about the tendency for the shorter period stars to increase their periods. This result comes mainly from $\omega$ Centauri and it can be seen in Figure 1 that there are large intervals when the W Virginis stars in this cluster were not studied.

Period changes for some W Virginis stars in the field have been studied by Kwee (1967). The longer period stars tend to have fluctuating periods, similar to those in the clusters. However, of the 3 short period stars, one has a decreasing period, one a fluctuating period and one a constant period, over an interval of about $40 \mathrm{yr}$.

At the present time, it appears that the W Virginis stars with periods greater than 8 days have periods which either remain constant or fluctuate randomly. The variables with periods less than 8 days have not yet been studied adequately. When more observations are available for $\omega$ Centauri, we hope to understand these stars more fully.

Bailey, S. I.: 1902, Harv. Ann. 38, 1.

\section{References}

Coutts, C. M. and Sawyer Hogg, H.: 1972a, Bull. Am. Astron. Soc. 4, 217.

Coutts, C. M. and Sawyer Hogg, H.: 1972b, in press.

Demers, S. and Wehlau, A.: 1971, Astron. J. 76, 916.

Dickens, R. J. and Carey, J. V.: 1967, Roy. Observ. Bull., No. 129.

Eggen, O. J.: 1961, Roy. Observ. Observ. Bull., No. 29.

Kraft, R. P.: 1972, in A. G. Davis Philip (ed.), The Evolution of Population II Stars, Dudley Obs. Reports No. 4.

Kwee, K. K.: 1967, Bull. Astron. Inst. Neth. Suppl. $2,97$.

Martin, W. Chr.: 1938, Ann. Sterrew. Leiden 17, pt. 2, 1.

Mengel, J. G.: 1972, IAU Colloq. No. 21, Toronto.

Osborn, W.: 1969, Astron. J. 74, 108.

Poole, L.: 1968, Master's Thesis, University of Toronto.

Sawyer, H. B.: 1931, Harv. Circ., No. 366.

Sawyer, H. B.: 1935, Publ. Dom. Astrophys. Obs., Victoria 6, 265.

Sawyer Hogg, H. and Wehlau, A.: 1966, Publ. David Dunlap Obs. 2, 451.

Sawyer Hogg, H. and Wehlau, A.: 1968, Publ. David Dunlp Obs. 2, 493.

Schwarzschild, M. and Härm, R.: 1970, Astrophys. J. 160, 341.

Strom, S. E., Strom, K. M., Rood, R. T., and Iben, I.: 1970, Astron. Astrophys. 8, 243.

Wilkens, H.: 1967, Mitt. veränderl. Sterne 4, 93. 


\section{DISCUSSION}

Wesselink: Have you noticed any changes in form of light curves?

Coutts: There seems to be, but the results are difficult to obtain because of differences in scales. Wehlau: A drop in amplitude over the years is seen in the Toronto observations for many variables in M14 and may be due to the gradual increase in sky background at Toronto.

Belserene: I have looked at V 60 and V 61 in $\omega$ Cen and have the impression that there are real variations in the shape of the light curves. Also, a very rapid period increase for $\mathrm{V} 92$ fits many of the epochs. I haven't looked at them all. The rate is about 10 cycles per million years. 